\title{
Proposal for a layer-based loT construction method and its implementation and evaluation on a rolling stand-up walker
}

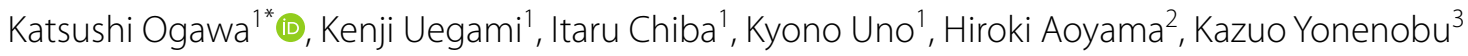 \\ and Seonghee Jeong ${ }^{1}$
}

\begin{abstract}
It is a problem that the poor gait function of many elderly people affects their activities of daily living (ADL), and deteriorates their quality of life (QoL). To enhance rehabilitation of the gait function, it is desirable to increase the amount of training received. In this research, we develop a measurement system for a gait training device that enables effective rehabilitation using the internet of things (loT) and measurement technology proactively, which aims to realize gconstant rehabilitation $\mathrm{h}$ by considering $\mathrm{ADL}$ (except training time) as rehabilitation. We propose an IoT measurement system model that classifies each function of the loT system in a hierarchical structure to promote systematic development more efficiently. Based on this model, we (1) modularize a device for measuring the upper limb load, acceleration, and gait distance, (2) develop a device for presenting the target value of rehabilitation visually to the user using the internet, and (3) implement the model on a commercial rolling stand-up walker and gait training device. We confirm that we can obtain excellent measurements using each mounted module.
\end{abstract}

Keywords: Gait training device, Convalescent rehabilitation, loT measurement system, Layer-based loT system

\section{Introduction}

Impairment of the gait function of the elderly affects their activities of daily living (ADL), and deterioration of their quality of life $(\mathrm{QoL})$. It is desirable to increase the amount of training to enhance the effect of rehabilitation of the gait function [1-3]. However, although the current number of active physiotherapists may seem satisfactory, in reality, it is insufficient as adequate rehabilitation cannot be provided to every patient [4]. Considering the current situation, the following are considered to be the most effective solutions to this problem :(1) evaluate rehabilitation quantitatively and implement it efficiently and effectively, and (2) carry out "constant rehabilitation," i.e., quantify the exercise information of the ADL and evaluate the effects of rehabilitation anytime and anywhere.

\footnotetext{
*Correspondence: ogawa@osakac.ac.jp

${ }^{1}$ Department of Electro-mechanical Engineering, Osaka Electro

Communication University, Osaka, Japan

Full list of author information is available at the end of the article
}

However, the evaluations of rehabilitation effects in gait training are mostly qualitative, as subjectively performed by therapists, and currently, it is common to train only for a fixed time owing to safety concerns. Additionally, the acquisition of exercise information is difficult to perform. A common support method for load-adjusting gait training at rehabilitation centers is the use of weight scales, where an affected leg is placed on the weight scale in a standing position, appropriate load is sensed, and walking is performed while remembering the sense. This method is simple as it occurs only in a stationary state, and is widely used in clinical settings because it allows the therapist to sufficiently check the load situation. However, since the load on the affected foot cannot be confirmed during walking, there is a problem in terms of safety; therefore, the training efficiency is usually low and there is a risk that an excessive load is mistakenly applied [5-7]. 
There are a few systems that provide the feedback of the target value of the load to the rehabilitation patient. For example, Sugiyama developed a system that used a foot pressure sensor to provide feedbacks to the patients about changes in the walking load using sound [8]. There is also a method that uses a sandal type load meter with a built-in load measuring sensor [9]. When the set value exceeds, a warning sound is emitted. The patient continues to increase the load until a warning sound is heard and trains to decrease the load on hearing the warning sound. In this method, the patient must wear dedicated sandals that can often feel cumbersome. Consequently, this method is rarely used in rehabilitation sites because the patient cannot train in daily walking conditions.

On the other hand, floor reaction force measuring instruments can quantitatively measure the amount of load placed on the lower limbs; however, they have limited installation locations. Not only are they unsuitable for measuring continuous movements such as walking, but also expensive and complicated to operate, limiting their use in general clinical facilities [10, 11]. Fukuyama developed a system that feeds back real-time information by displaying the measured value on the front display at the place where the floor reaction force gauge is installed [12]. A walking support device (robot) that can measure motion information during walking has also been proposed $[13,14]$; however, the measuring device is incorporated in a dedicated main unit that makes it difficult to introduce it to the rehabilitation sites. All these methods require a special device to be worn on the body or largescale dedicated device and are not suitable for continuous monitoring at home or during ADL.

To address these shortcomings, we developed a system based on a walker so that the patient can use it every day without wearing special equipment on the body. The system enables high reproducibility of rehabilitation by visually feeding the measured values such as upper limb load to the rehabilitation trainer in real-time with numerical values and graphs. We also propose a construction method that uses a hierarchical structure model of an internet of things (IoT) measurement system that provides quantitative data of the rehabilitation effect to patients and physiotherapists through multimedia. Our aim is to provide visualization of the exercise information during gait training and realize gconstant rehabilitation, $h$ i.e., rehabilitation in ADL in addition to dedicated training time with physiotherapists.

\section{Concept}

We developed a gait training measurement system that solves the problems of conventional methods for patients who require gait training for spinal and lower limb disease. In order to solve these problems, a system that satisfies the following conditions was required: (1) the patient is not burdened, (2) the load during walking can be measured, and (3) the system has a feedback function that allows the patient to apply the appropriate load.

Specifically aimed at its practical introduction to real rehabilitation work sites, the measuring device that can be attached to many walking assist devices was modularized to enhance versatility and convenience. As a result, exercise information can be measured by attaching it to a walking assistance device that is used at home in ordinary rehabilitation and daily life. This system makes it possible to provide feed back of the quantified and visualized information to the patient, which enables effective gconstant rehabilitation $\mathrm{h}$.

In our previous research, we carried out modularization of the measuring instrument and evaluated it incorporating the prototype into a KA-391 [15] manufactured by Paramount Bed [16]. An external view of the robotics exercise walker is shown in Fig. 1. Additionally, training was conducted using the prototype, and as a result, it was found that the upper limb dependent load during walker training could be represented using a numerical index that is gradually reduced as the patient gains independence [17]. Constant rehabilitation that can provide and improve motivation for rehabilitation at home can also be expected to continue daily after the patient is discharged from the hospital.

The aims and approach of this research are summarized as follows: (1) modularize the measuring instrument to allow installation on a commercially available walker, (2) introduce IoT to visualize data centrally managed in the cloud, and (3) measure exercise information constantly from daily training to daily life. The proposed system is characterized by the ability to evaluate exercise information and confirm the effect of rehabilitation anytime

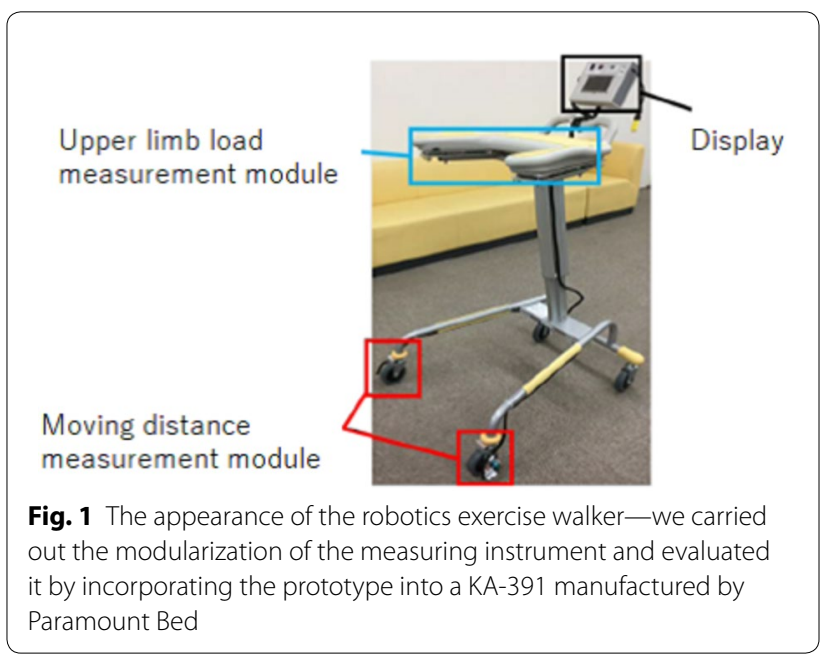


and anywhere, because there is a device and measurement system that can be used for rehabilitation, which is close to the patient at home. Moreover, it possesses the feature that it is not necessary to attach any additional equipment other than the equipment being used daily. Additionally, for therapists, the evaluation criteria can be clarified and streamlined using the automated data management component and quantification of the exercise information. As a result, the device has the feature that allows the both therapist and patient to concentrate on the treatment.

\section{Implementation of the loT system to a rolling stand-up walker IoT measurement system model (IMSM) proposal}

To measure and manage exercise information continuously during rehabilitation training time, IoT technology was introduced to the constructed measurement system. Therefore, to promote system development more efficiently, we propose the IoT measurement system model (IMSM) in which each function of the IoT system is classified in a hierarchical structure (Fig. 2). The aim of this proposal is to clarify each information processing task and system structure. When developing an IoT system, it is necessary to develop the optimal solution that takes into consideration the system's performance, processing speed, and part costs. Simultaneously, considerable effort is required to identify technical issues and bottlenecks in the data processing. Thus, there are many factors that impede development. Our philosophy is that a more efficient system development is possible if the approach to finding the optimal solution is organized while attempting to realize the envisioned system. One of the obstacles in achieving this goal is that the definition of an IoT system may be different for each person; therefore, the idea of how to execute each process or function in the system is likely to be different for each person. Therefore, it was decided that it would be better to define common classifications of IoT systems and establish a development method based on those classifications. The IMSM was subsequently defined by organizing each function and process in layers and then organizing the connections of each layer. To construct the optimal system more simply, it was decided to perform classifications in each layer based on their measurement data flow. This approach is the foundation of the proposed IMSM. Using this method to build the system, each task of sensing, data communication, and data processing was clearly organized and indicated, and information sharing was smoothly performed during system solution development among the multiple member efficiently and effectively.

As shown in the Fig. 2, there are five levels of classification: Layer 1 contains the measurement hierarchy of the physical data recorded by each sensor. Layer 3 contains the conversion processing hierarchy of the raw data measured by the sensors to exercise data. Layer 5 comprises exercise data accumulation and analysis hierarchy. Layers 2 and 4 are communication layers connecting the other layers.

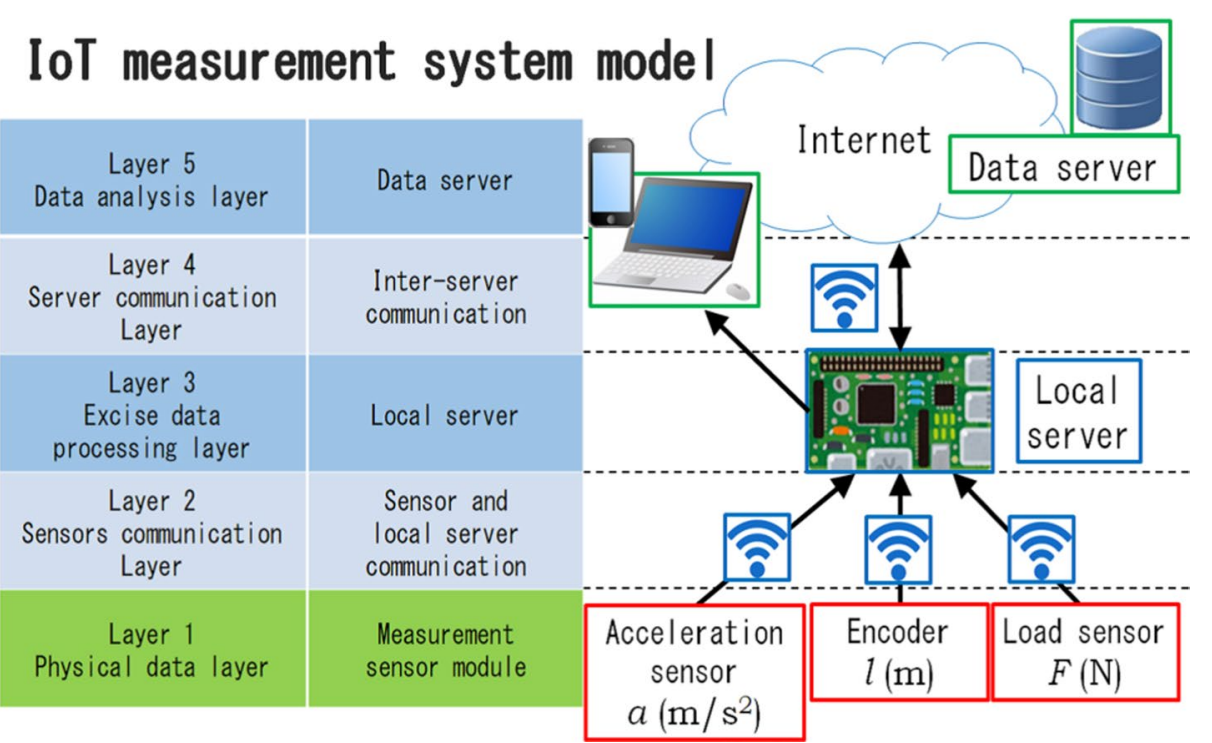

Fig. 2 loT measurement system model (IMSM) — to construct the optimal system more simply, classification is performed in a layer-based approach using the data flow from the sensor's measurement 


\section{Construction of the measurement system}

We constructed the measurement system of the IoT rolling stand-up walker that was based on the proposed IMSM. This system consisted of a modular measuring instrument mounted on a commercial walker. With the mechanism shown in Fig. 3, it is known from our previous research that the upper limb dependence weight in gait training with a walker is a numerical index that is reduced as the patient gains independence during convalescent-phase rehabilitation [17]. When an upper limb load $\left(W_{U}\right)$ is applied to the walker armrest, the lower limb load $\left(W_{L}\right)$ is reduced. This is because the relationship between the load applied to each limb and the total weight $\left(W_{T}\right)$ follows the behavior described in Eq. (1). It is possible to quantitatively adjust the upper limb load $\left(W_{U}\right)$ measured by the load sensor while looking at the graph and the target value on the user interface (UI) display attached to the walker, and therefore, quantitatively adjust the lower limb load.

$$
W_{L}=W_{T}-W_{U}
$$

We installed load sensors on the left and right armrests so that we could check the balance of the upper limb loads, i.e., $m_{l}$ (left), $m_{r}$ (right), and $W_{U}$ (upper limb load), all in units of $\mathrm{kg}$. The relationship between each parameter value is shown in Eq. (2). We installed distance and acceleration measurement sensors to measure the motion information of the patient's gait, where the moving distance in the anteroposterior direction was $l_{y}(\mathrm{~m})$ and the acceleration of the armrests in the anteroposterior direction was $a_{y}\left(\mathrm{~m} / \mathrm{s}^{2}\right)$.

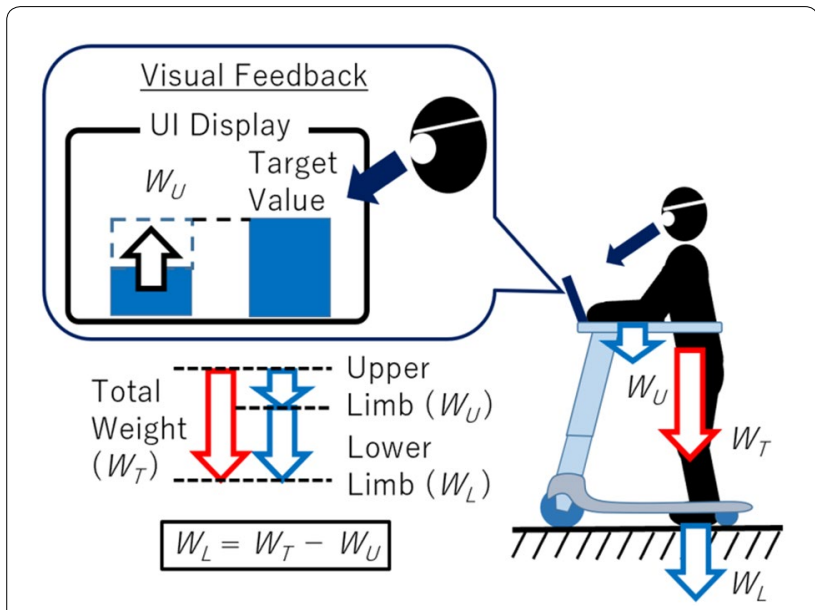

Fig. 3 When $W_{U}$ is applied to the walker, $W_{L}$ is reduced following the relationship between the load applied to each limb and $W_{T}$, as given in Eq. (1). It is possible to quantitatively adjust the loads measured by the load sensors while looking at the graph and target value on the UI display attached to the walker

$$
W_{U}=m_{l}+m_{r}
$$

Applying this mechanism in the context of the IMSM, we developed appropriate modules for each layer and selected the appropriate communication methods. In other words, each sensor module suitable for this system was developed in the physical-data layer (Layer 1 ), and a sensor network that enabled each module to send and receive appropriate data was constructed in the sensor-communication layer (Layer 2). In the exercise-data-processing layer (Layer 3 ), the system had a local data server that collected the measurement data from the sensor modules. In the server-connection layer (Layer 4), the walker was constantly connected to an in-hospital LAN and performed data communication. In the data-analysis layer (Layer 5), we set up a data file server to manage the rehabilitation training data and exercise information of daily life.

Fig. 4 shows the measurement system built based on the IMSM, and Fig. 5 shows the conceptual system diagram of the IoT rolling stand-up walker. In Layer 1 , sensor selection and the measurement method are the important factors. The measurement sensors that included (1) an upper limb weight measurement sensor, (2) acceleration sensor, and (3) a device for measuring the movement amount in the anteroposterior direction were mounted in this system. To realize modularization of the sensor system, we also developed a highly versatile printed electronic circuit board (PCB) that supported wireless communication and wired serial communication that is compatible with various input methods shown in Fig. 6a. In the next section, we will elaborate on this PCB.

In Layer 2, we can select the optimal communication method for the sensor system. This allows the rehabilitation training to proceed unrestricted, and the system is simple. The communication protocol requires highspeed, real-time measurement at $50 \mathrm{~Hz}$ to measure motion information; therefore, we adopted UDP communication using Wi-Fi.

Layer 3 receives communication data from Layer 2 that accumulates and visualizes the data and generates a CSV file. Access occurs via Wi-Fi for each module, and a tablet terminal is used to visualize the exercise information. Herein, we used a Raspberry Pi $3 \mathrm{~B}+$ as the data server that converted the measured data sent from each measuring device into exercise information and stored the data and at the same time communicated with the outside. We used Node RED for the web server and data visualization, and MYSQL for the database. Exercise values can be confirmed by web access from an external smartphone or tablet terminal. 
System design based on loT measurement system model

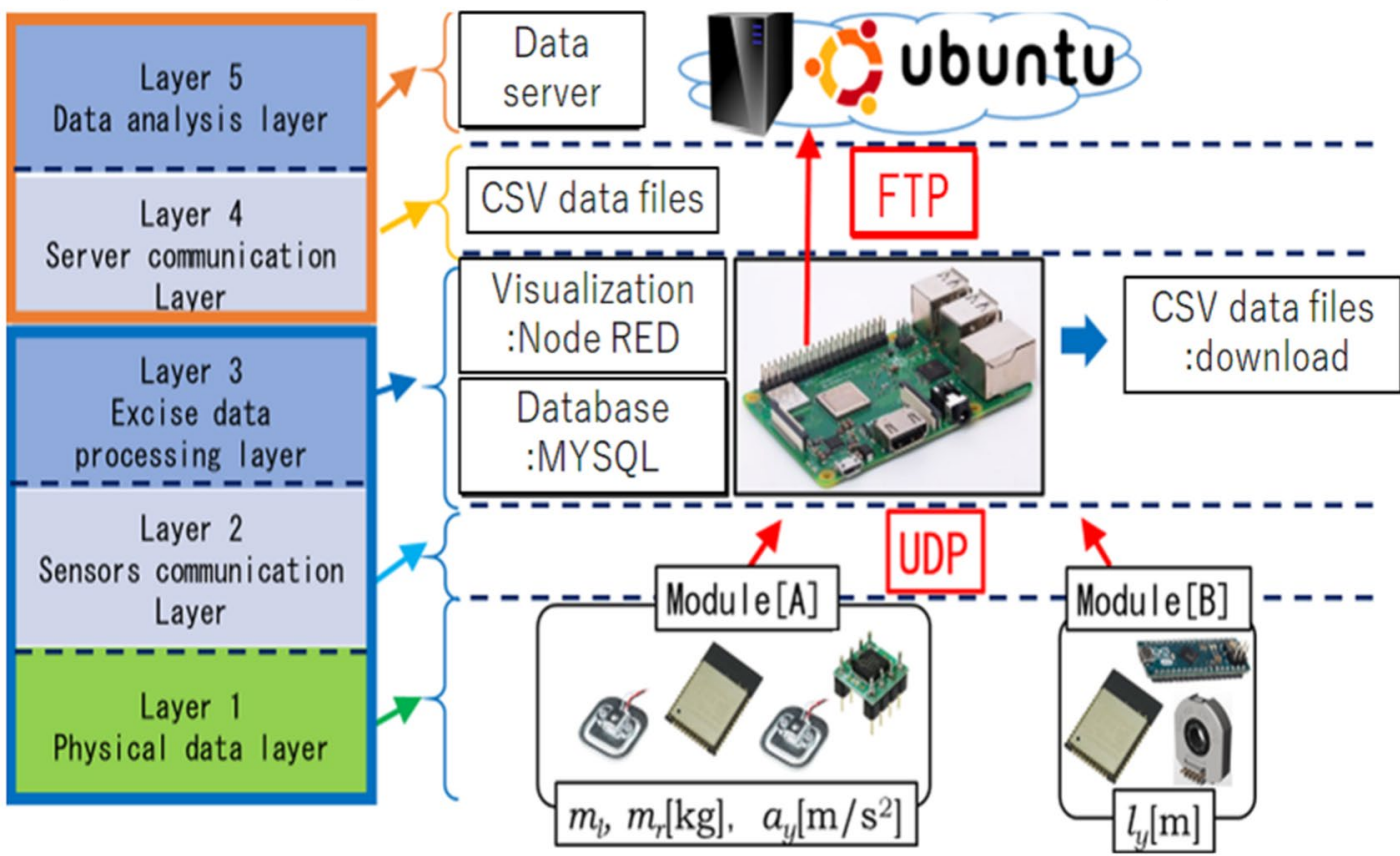

Fig. 4 Measurement system based on the IMSM that was implemented on the rolling stand-up walker —each sensor system was modularized to allow access using the server in the Layer 3 using UDP over Wi-Fi, user rehabilitation CSV data files were transferred in the Layer 5 to the server using FTP

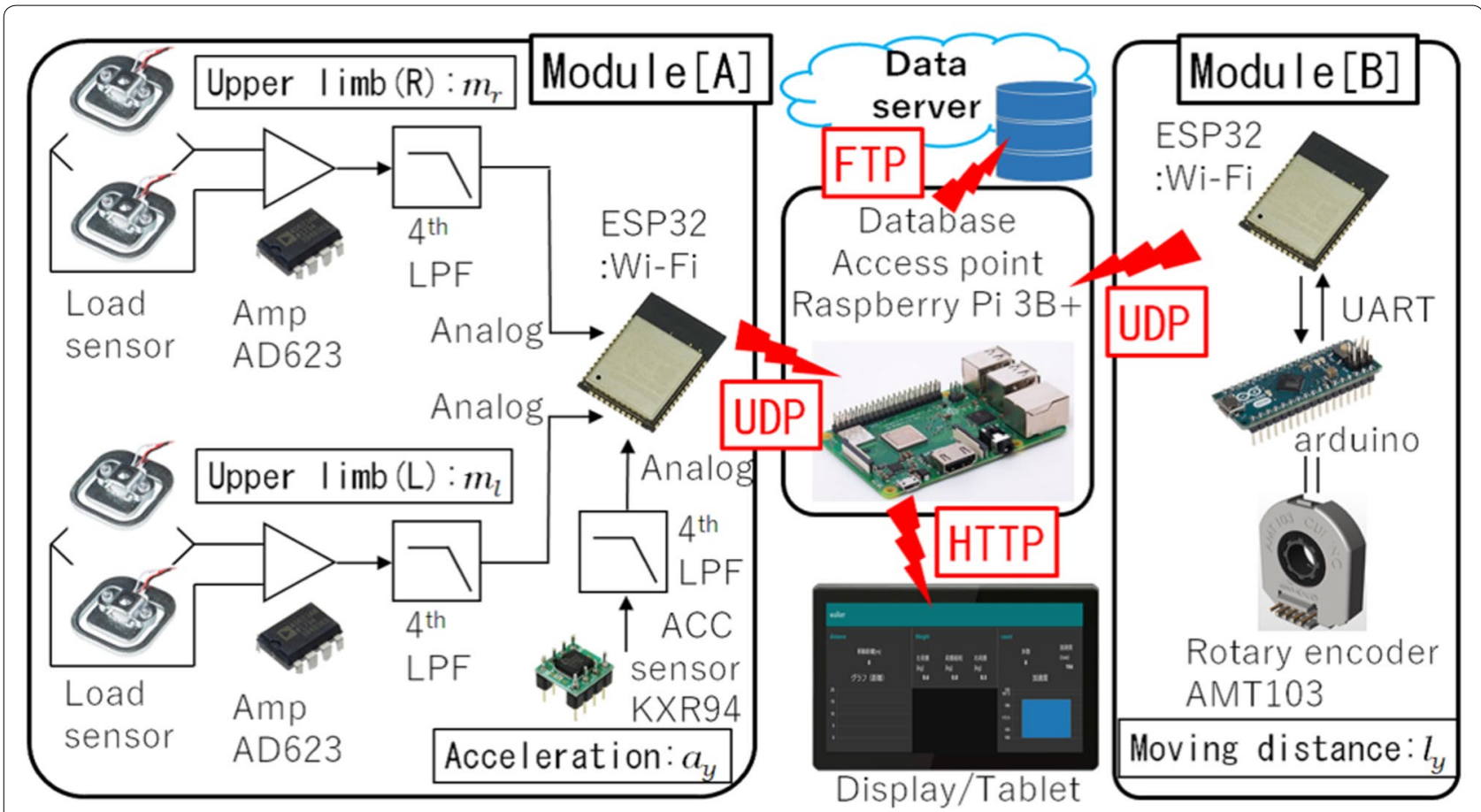

Fig. 5 Conceptual system diagram of the rolling stand-up walker measurement system —-the Module [A] has left and right load sensors ( $\left.m_{l}, m_{r}\right)$ and acceleration sensors $\left(a_{y}\right)$, and Module $[B]$ has a rotary encoder for measuring the distance $\left(I_{y}\right)$ 


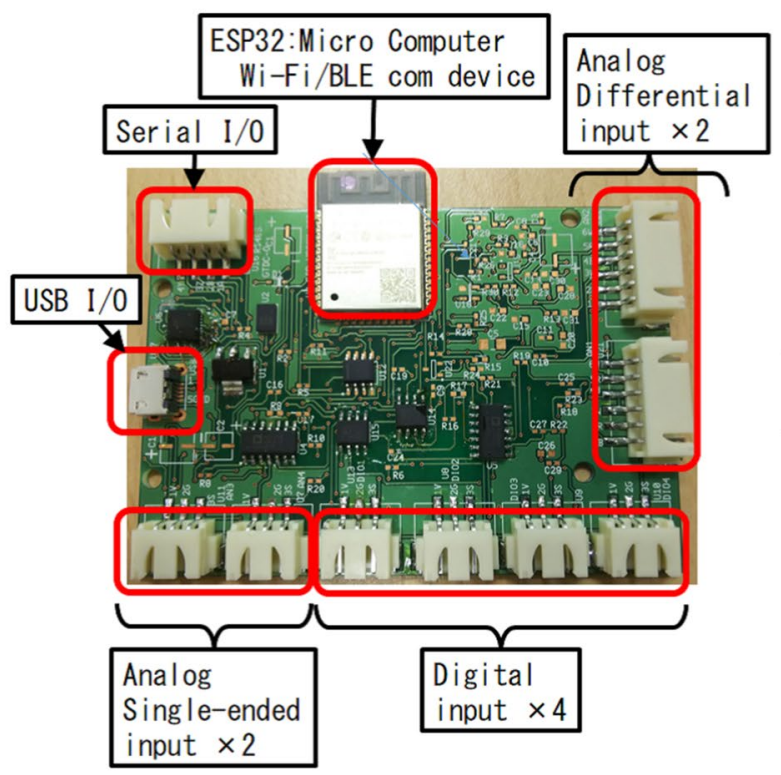

(a) PCB appearance

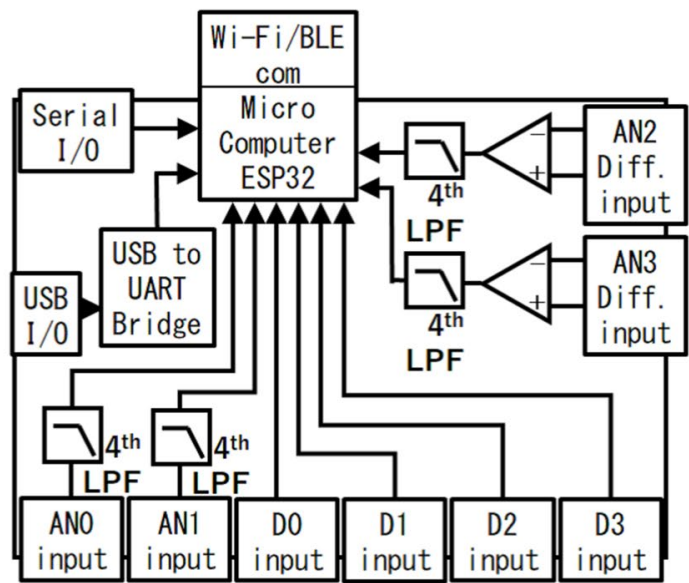

(b) Block diagram

Fig. 6 A PCB appearance and block diagram a A PCB design and development to modularize the measurement sensor system -in the walker, it is used universally for both the Modules A and B, b PCB circuit block diagram in which an ESP32 microcomputer was used -it has Wi-Fi and Bluetooth communication functions, an AD converter, GPIO, and other functions

Figure 7 shows a screenshot of the tablet touch panel display as seen in the internet browser. A graph showing the total upper limb weight and rehabilitation target value are displayed in real-time for visual feedback. Other measurement values such as the left and right upper limb loads, movement distances, and acceleration values are also displayed in real-time. Users can perform quantitative rehabilitation while looking at this panel on the armrest, and the user operates the system with buttons on the display, i.e., operations for starting/ending measurement, generating a CSV file, resetting the distance measurement value of the Module $B$, and inputting personal information, are provided. The physiotherapist can quantitatively evaluate the effect of the rehabilitation by viewing the same screen on another tablet.

In Layer 4 the CSV files are transferred to the data file server in the hospital LAN in the Layer 5 using FTP communication using Wi-Fi. In the Layer 5, user rehabilitation data files received using FTP from the Layer 4 are stored in association with personal data registered in advance. These data are used as an index of the rehabilitation effect and recovery and confirmation of daily activities. Fig. 8 shows the appearance sensor placement in the rolling stand-up walker. Module A that was equipped with load sensors and an acceleration sensor was mounted under the armrest. The load sensor was set below the position where the arm was placed to measure the left and right upper limb loads. The PCB to which the acceleration sensor was connected for data measurement and communication was set in the center of the armrest. Module B that had a rotary encoder to measure movement distances arising from linear motion was attached to the front wheel. Respective flowcharts of the firmware of Modules A and B are shown in Figs. 9, 10. In Module $\mathrm{A}$, at first configure the system settings. Read the analog value of the acceleration sensor and calculate from the obtained value. Next, it reads the analog value of the load sensor and calculates the left and right load values and the total upper limb load value. Then, send the UDP data, and when the transmission is completed, return to reading the sensor value. In the Module $B$, after setting the system, the value of the rotary encoder is read by the arduino, and the distance is calculated from the value. The obtained data is transmitted to ESP32 by UART communication. ESP32 performs data transmission by UDP communication, and returns to reading of sensor value when completed.

\section{Development of a versatile PCB}

As shown in the Fig. 6b, we developed a PCB that was mounted with 4th-order low-pass filter and amplifier circuits to measure each physical quantity using each sensor. The PCB had an onboard microcomputer to execute signal processing as well as wireless and wired communication capabilities. The PCB was constructed using an adopted microcomputer ESP32 that had a wireless 


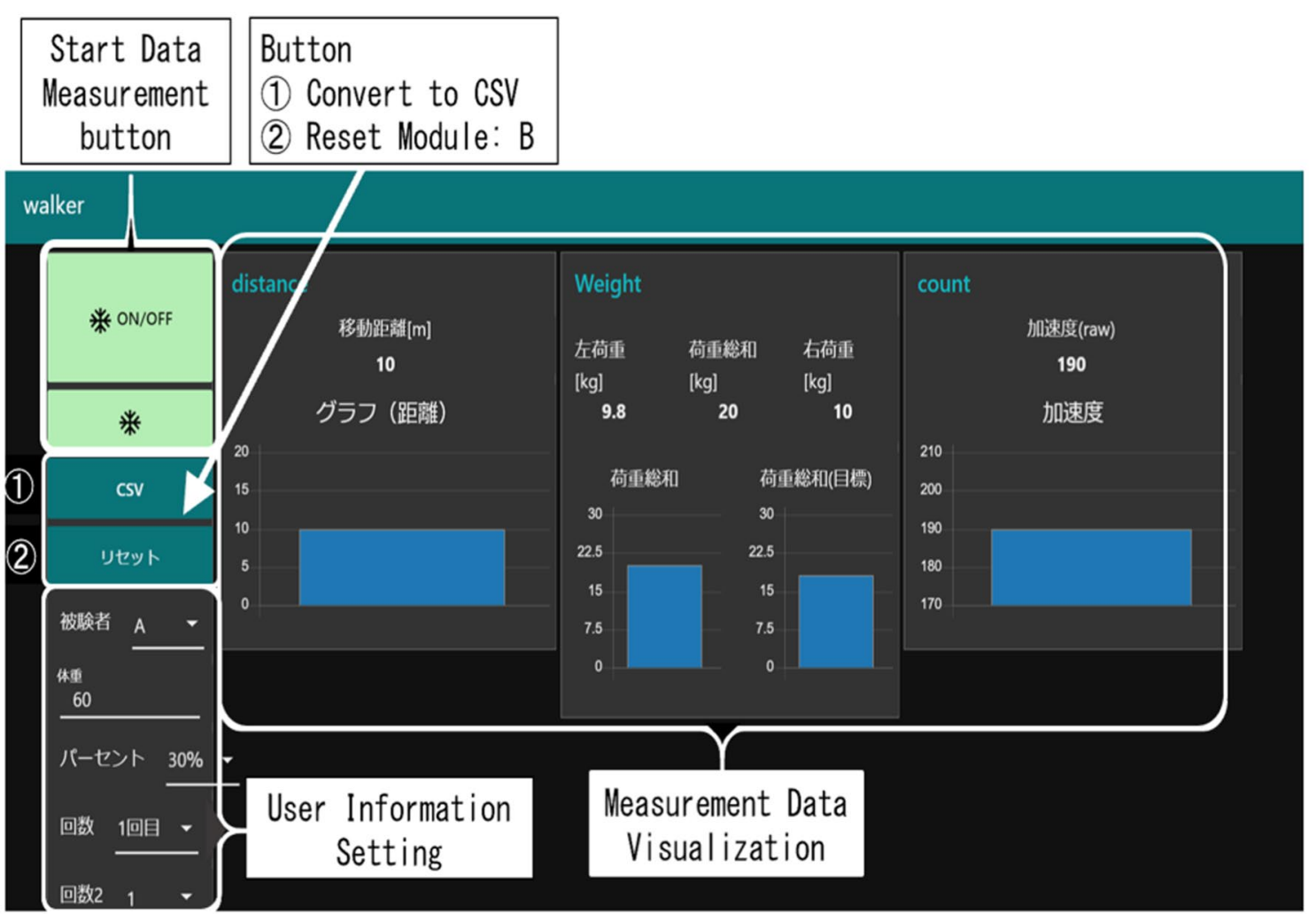

Fig. 7 UI screen displays as seen on the internet browser-the UI shows the moving distance $I_{y}$ and its integrated graph, the upper limb weights $m_{1}, W_{T}$, and $m_{r}$, the acceleration $a_{y}$, and its magnitude graph
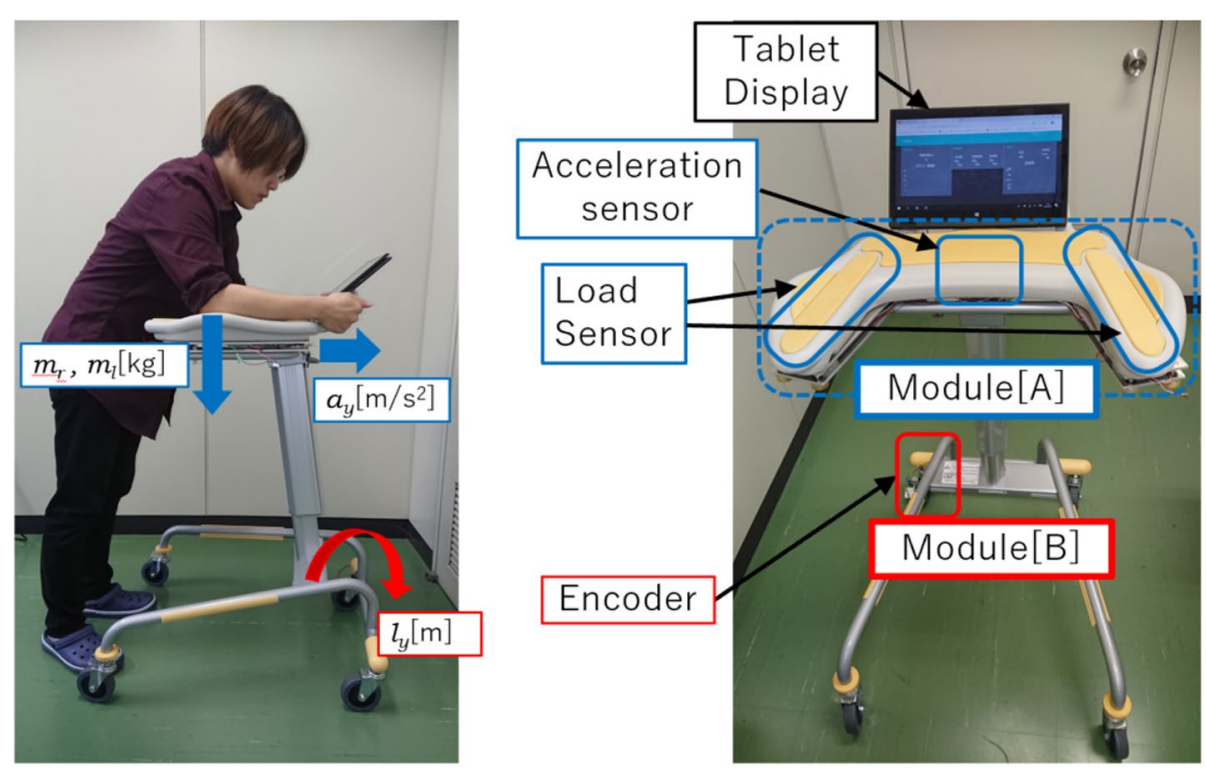

Fig. 8 The appearance of the rolling stand-up walker and sensor placement-access was achieved using Wi-Fi from each module and the tablet terminal to realize system visualization of the exercise information 

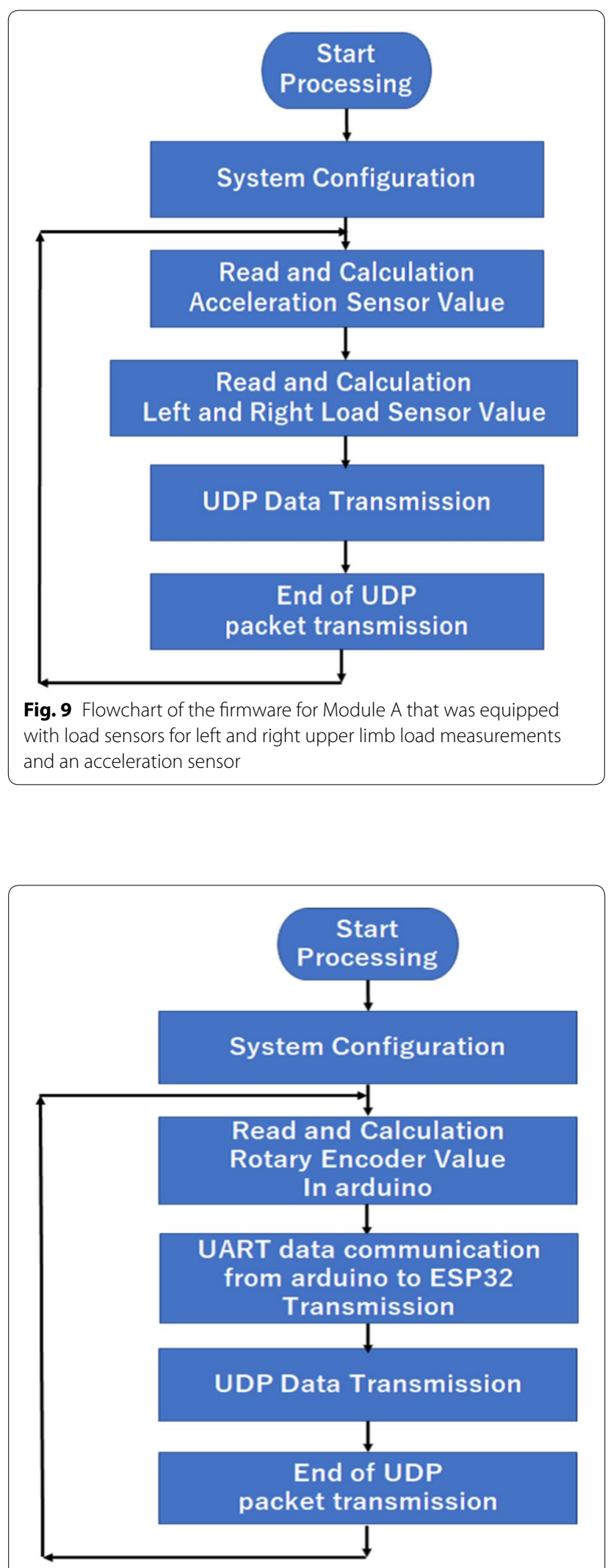

Fig. 10 Flowchart of the firmware for Module B that had a rotary encoder for obtaining distance measurements of linear motion module which could support Wi-Fi and Bluetooth. It had four channels of analog input ports and four channels of digital input ports. Two analog ports were differential input type and the other two were single-ended input type. It had a wired serial communication $\mathrm{I} / \mathrm{O}$ port.

System implementation of the Modules A and B was performed using the same PCB. In Module A, two channels of differential input ports were used for the left and right load measurements. A bridge circuit with a set of two load sensors (one channel each) was constructed, and the differential voltage was input to the analog port of the microcomputer through a 4th-order low-pass filter and an amplification circuit. Additionally, one channel consisting a single-ended analog input port was used to measure acceleration. In Module B, processing of the two-phase pulse count of the rotary encoder was performed using an arduino micro, and data communication was performed using the serial $\mathrm{I} / \mathrm{O}$ port.

\section{Evaluation of the loT rolling stand-up walker Evaluation method}

To verify the operation and practicality of the developed measuring device, the same verification test as performed for walking training in actual rehabilitation was conducted. The subjects were nine healthy men and women aged in their 20s. This study was carried out with the approval of the Osaka Electro-Communication University Ethics Committee (Approval Number: 19-003), and the subjects of the study were informed in advance and they agreed to be tested. In this verification test, each sensor measurement value collected in Layer 3 was displayed on the browser using the internet to the person who performed the rehabilitation and visualization of the rehabilitation activity was realized. A graph showing the total upper limb weight and the rehabilitation target value were displayed in real-time for visual feedback, as shown in Fig. 3. Since the subject could confirm the rehabilitation effect by checking the measurement values and graph, it was expected that effective rehabilitation could be realized.

During the verification tests, each subject walked $3 \mathrm{~m}$ using the developed gait training device. We measured the relevant values using the IoT measurement system. The upper limb load measurement values were displayed in real-time using numerical values and graphs. Simultaneously, the target value of the upper limb load was displayed in the same manner. The subjects could check the graph while walking. They could also adjust the upper limb load via visual feedback, i.e., the load on the lower limb could be adjusted quantitatively. The target value was defined as the ratio of the upper limb load $\left(W_{U}\right)$ to the body weight $(B W)$ as $r U W(\%)$ : 


$$
r U W(\%)=\frac{W_{U}}{B W} \times 100
$$

In the experiment, the target $r U W$ values were $0 \%, 30 \%$, and $40 \%$. For the condition of $0 \%$, the test subjects simply put their upper limbs on the walker, and were in a state of walking by pushing the walker. Under the conditions of $30 \%$ and $40 \%$, the subjects were in a state of walking with an upper limb load applied to the walker and a reduced lower limb load. The measurements were performed three times for each upper limb load target value $r U W$. The subject was instructed to walk while adjusting the upper limb load to match the target value $r U W$ graph while viewing the upper limb load $W_{U}$ graph displayed on the tablet placed on the armrest. The height of the armrest was adjusted to the position of the elbow bent by each subject while standing.

Next, we conducted an experiment to confirm the correlation between the upper and lower limb load with the developed device and force plates on the floor (BP400600-2000, AMTI, USA). The subjects walked on the first of the three force plates with their right foot, and were careful not to place the wheels of the walker upon them. The walkway in the experimental room was approximately $4 \mathrm{~m}$ long, and to acquire data using a floor reaction force gauge, the walking distance in this experiment was set to $3 \mathrm{~m}$, i.e., we excluded data from the beginning and end of the walking sequence. We started to record the data after the declaration that they had learned the sensation of their target load through some pre-trial.

The measurement item was the maximum vertical ground reaction force per body weight (GRF/BW). A gait cycle that was selected for the data analysis comprised the first initial contact of the right foot on the first force plate to the second initial contact of the right foot. We determined that the representative value was the average from three trials in each condition. To confirm the reproducibility of measurement in humans, the average upper limb load of $3 \mathrm{~m}$ gait using the developed walker for each subject was calculated, and the interclass correlation coefficient (ICC $(1,3)$ ) was used. Compared with normal walking, each subject was measured using a walker under conditions that mimicked disease. For example, when joint destruction had progressed due to osteoarthritis, there may be a problem with the fitted artificial limb, resulting in a difference in each leg's length. We mimicked this by having the patient walk with a $4 \mathrm{~cm}$ block attached to their right sole, as shown in Fig. 11. In this case, there was a physical difference between the left and right leg length. These measurements were performed by five subjects among nine who usually performed walking measurements.

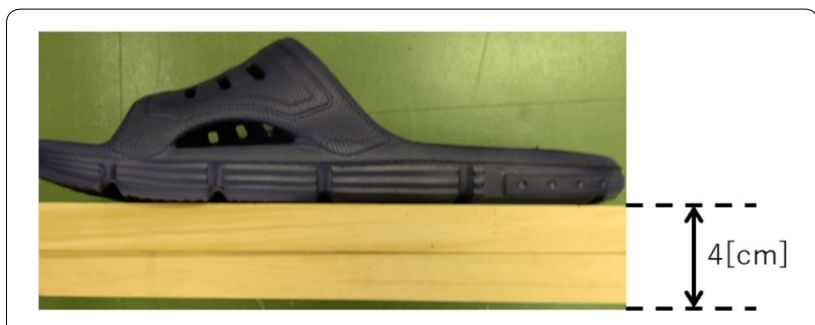

Fig. 11 The footwear with an attached block of $4 \mathrm{~cm}$ height on the sole of the right foot-this allowed us to make measurements of asymmetric walking

Table 1 Maximum vertical GRF/BW

\begin{tabular}{|c|c|c|c|}
\hline ruW & $0 \%$ & $30 \%$ & $40 \%$ \\
\hline $\mathrm{GRF} / \mathrm{BW}(\mathrm{SD})^{\mathrm{a}}$ & $95.7(8.4)$ & $69.6(5.2)$ & $62.2(9.0)$ \\
\hline
\end{tabular}

Values represent mean in the each condition (\%)

Standard deviation (SD)

a Statistically significant difference between conditions are noted for $\alpha=0.01$

To evaluate the visual effect of the upper limb load with the developed walker, $30 \%$ and $40 \%$ tasks were performed without visual feedback to the subjects. The measurements were performed three times, and the upper limb load data for a $3 \mathrm{~m}$ gait was collected. The measurement procedure was as follows. Without the tablet display on the walker, the subject did not know their upper limb load measurement results. The subjects gradually increased the upper limb load and simultaneously, the other person told the patient that the target value had been reached verbally while watching the measurement results. The subjects memorized the state of each upper limb load. Then, after they released their upper limb load and waited about five seconds, measurement started again. In this experiment, measurements with $0 \%$ $r U W$ were not performed because $0 \%$ is required for the patient to only push with their upper limbs on the walker, which was less relevant for visual feedback. These measurements were performed by five subjects among the nine who normally performed walking measurements.

\section{Measurement result and consideration Maximum vertical GRF/BW}

The maximum vertical GRF/BW showed a linear reduction as the $r U W$ value increased (Table 1). The sum of the $r U W$ and GRF/BW was near 100 percent, except for $0 \% r U W$. There were significant differences between all the conditions $(\mathrm{p}<0.01)$.

\section{ICC and reliability}

Table 2 shows the measurement results of the average upper limb load ratio for normal walking conditions for 
Table 2 Average of rUW at each target value in normal walking conditions

\begin{tabular}{lrll}
\hline (\%) & \multicolumn{3}{l}{} \\
\hline & \multicolumn{1}{c}{$\mathbf{0 \%}$} & $\mathbf{3 0 \%}$ & $\mathbf{4 0 \%}$ \\
\hline A & $2.6 \pm 0.1$ & $28.8 \pm 0.1$ & $38.0 \pm 1.3$ \\
B & $3.3 \pm 0.4$ & $26.5 \pm 1.0$ & $31.7 \pm 0.8$ \\
C & $20.5 \pm 3.0$ & $25.7 \pm 0.4$ & $31.1 \pm 0.3$ \\
D & $0.7 \pm 1.0$ & $26.0 \pm 0.6$ & $33.6 \pm 0.5$ \\
E & $4.2 \pm 0.9$ & $29.4 \pm 0.3$ & $38.4 \pm 1.2$ \\
F & $8.5 \pm 0.2$ & $29.2 \pm 0.7$ & $38.2 \pm 1.8$ \\
G & $7.4 \pm 1.1$ & $29.7 \pm 0.9$ & $39.0 \pm 1.2$ \\
H & $5.8 \pm 1.6$ & $28.6 \pm 0.6$ & $37.0 \pm 0.8$ \\
I & $12.8 \pm 0.6$ & $29.0 \pm 0.5$ & $39.7 \pm 0.8$ \\
\hline
\end{tabular}

$\mathrm{n}=9$ Average \pm standard deviation

Table 3 ICC at each target value in normal walking conditions

\begin{tabular}{lll}
\hline Target value & ICC (1,3) & $\mathbf{9 5 \% ~ C l}$ \\
\hline $0 \%$ & 0.97 & $0.89,0.99$ \\
$30 \%$ & 0.96 & $0.84,0.98$ \\
$40 \%$ & 0.97 & $0.87,0.98$ \\
\hline
\end{tabular}

ICC Intraclass Correlation Coefficient

95\% Cl 95\% Confidence Interval

$r U W$ values of $0 \%, 30 \%$, and $40 \%$. For each $r U W$ value, the intraclass correlation coefficient (ICC) was 0.8 or more (Table 3 ). These results indicate that the measurement reliability of the upper limb load with the developed walker was good.

For the rUW measurement results, individual differences for each subject were observed. There was a particularly large individual difference at the target value of $0 \%$. It is inferred that each subject had a different interpretation of achieving the target value. Specific attention was paid to the Subjects $C$ and I who were very far off at $0 \%$. Subject $C$ was significantly higher at $0 \%$, but significantly lower at $30 \%$ and $40 \%$. In other words, it is presumed that this is a recognition that the target achievement may be ambiguous. On the other hand, Subject I showed relatively accurate values at $30 \%$ and $40 \%$. In other words, only $0 \%$ measurement may have a reduced awareness of accuracy. For both subjects, the standard deviation showed a relatively low value. Considering these facts, the subjects' perception and techniques for handling a walker are reflected in the measurement values, and it is considered that the measurement system is highly reproducible. Therefore, it is considered that these individual differences in the measured values can be improved via training.
Table 4 Average of rUW at each target value for the asymmetric leg length condition

\begin{tabular}{llll}
\hline (\%) & \multicolumn{3}{l}{} \\
\hline & $\mathbf{0 \%}$ & $\mathbf{3 0 \%}$ & $\mathbf{4 0 \%}$ \\
\hline E & $4.4 \pm 0.3$ & $29.3 \pm 0.5$ & $39.3 \pm 0.4$ \\
F & $7.0 \pm 0.4$ & $28.4 \pm 1.4$ & $35.7 \pm 1.1$ \\
G & $5.9 \pm 0.6$ & $29.7 \pm 0.8$ & $38.5 \pm 0.6$ \\
H & $9.7 \pm 0.5$ & $29.3 \pm 0.9$ & $37.9 \pm 0.3$ \\
I & $17.7 \pm 4.2$ & $33.8 \pm 0.2$ & $41.9 \pm 1.5$ \\
\hline
\end{tabular}

$\mathrm{n}=5$ Average \pm standard deviation

Table 5 ICC at each target value for the asymmetric leg length condition

\begin{tabular}{lll}
\hline Target value & ICC (1,3) & $\mathbf{9 5 \% ~ C l}$ \\
\hline $0 \%$ & 0.95 & $0.52,0.99$ \\
$30 \%$ & 0.94 & $0.46,0.99$ \\
$40 \%$ & 0.94 & $0.46,0.99$ \\
\hline
\end{tabular}

ICC Intraclass Correlation Coefficient

95\% Cl 95\% Confidence Interval

Table 4 shows the measurement results of the upper limb load ratio average in the asymmetric leg length condition for $r U W$ values of $0 \%, 30 \%$, and $40 \%$. Despite the asymmetric foot condition, as seen for the normal walking conditions, the ICC values were 0.8 or more (Table 5). This result indicates that the measurement reliability of the condition assuming lower limb disease was good.

As an evaluation of the practicality of the developed walker, we measured the amount of upper limb load during walking of healthy subjects, and as a result, we were able to obtain high reliability. We believe that high reproducibility can be a factor in high rehabilitation effects. Humans are different from machines, and it is difficult for humans to exactly repeat the same movements even when measured under the same conditions. Thus, it is difficult to increase reproducibility with high reliability in the actions performed by humans. However, the excellent reproducibility measured herein suggests that this walker can be used as a gait-evaluation tool at rehabilitation centers. Therefore, it is important to verify why the reproducibility was so high. Now, let us consider the reason for this high reproducibility.

Table 6 shows the average $r U W$ measurement results in the gno tablet display h condition for $r U W$ values of $30 \%$ and $40 \%$. The ICCs of the target value of $30 \%$ were 0.8 or more (Table 7 ), but the ICCs for a target value of $40 \%$ were less than 0.8 . These results indicate that the measurement reliability of no visual feedback was not 
Table 6 Average of rUW at each target value in the "no tablet display" condition

\begin{tabular}{lll}
\hline (\%) & & \\
\hline & $\mathbf{3 0 \%}$ & $\mathbf{4 0 \%}$ \\
\hline E & $31.7 \pm 1.9$ & $41.5 \pm 3.2$ \\
$F$ & $33.5 \pm 0.4$ & $37.4 \pm 2.5$ \\
G & $29.7 \pm 1.0$ & $35.7 \pm 5.8$ \\
$H$ & $28.9 \pm 1.7$ & $35.2 \pm 1.2$ \\
I & $33.8 \pm 1.2$ & $37.0 \pm 1.3$ \\
\hline
\end{tabular}

$\mathrm{n}=5$ Average \pm standard deviation

Table 7 ICC at each target value in the "no tablet display" condition

\begin{tabular}{lll}
\hline Target value & ICC (1,3) & $\mathbf{9 5 \% ~ C l}$ \\
\hline $30 \%$ & 0.86 & $-0.23,0.97$ \\
$40 \%$ & 0.59 & $-2.64,0.91$ \\
\hline
\end{tabular}

ICC Intraclass Correlation Coefficient

95\% Cl 95\% Confidence Interval

good. Compared with other conditions, the standard deviation value was also large, showing that the measurement results were uneven and varied widely, and the reproducibility was low. It is suggested that the visual feedback system contributed greatly to the reproducibility of the measurement in this system. During the measurements, there were several subjects who said that they were uncomfortable because their upper limb load was unknown. We believe that rehabilitation when the patient is anxious cannot be expected to be highly effective. If the patient can rehabilitate with confidence, it can be expected to lead to high motivation for training.

\section{Measuring the walking state}

The measurement results of the demonstration test conducted under normal and asymmetric walking conditions for each $r U W$ target value are shown in the Figs. 12, 13, respectively. These measurements were performed for the subject $\mathrm{H}$. Each Figure shows graphs of the moving distance $l_{y}$, upper limb weight $m_{l}, m_{r}$ and $W_{U}$ and the acceleration $a_{y}$. Fig. 12a shows the measurement results under normal walking conditions using the walker with an $r U W$ target value of $30 \%$, and Fig. 12b shows the results under the asymmetric walking condition with an $r U W$ target value of $30 \%$. Fig. 13a shows the results under normal walking conditions with an $r U W$ target value of $40 \%$, and Fig. 13b shows the results under the asymmetric walking condition with an $r U W$ target value of $40 \%$.

Next, let us consider the results under each condition. In the upper limb weight graph for normal walking, it is thought that the waveform peaks of the left and right upper limb weights appear alternately because the center of gravity moves to the left and right, and back again, during walking. During this movement, the acceleration also greatly changes, and the walking motion can be read from the waveform. It can also be seen that the wave peaks and valleys of acceleration are regularly arranged, which respectively represent the positive acceleration applied to the walker when the foot steps from the ground, and the negative acceleration that is applied when the foot is placed on the ground. The number of steps can be measured counting the number of acceleration peaks. There is a characteristic in the waveform of the acceleration in the traveling direction, as shown in Figs. 12, 13, where the timing of landing at the heel (heel strike) and taking off at the toe (toe off) can be confirmed from the peaks. The walking cycle time can also be found from the acceleration waveform data. On the other hand, the step length and walking speed can be calculated from the moving distance data. Generally, all the data useful for efficient rehabilitation can be obtained using the device.

For the asymmetric walking data, high peaks appear at regular intervals in the upper limb weight graph. In terms of acceleration, a pair of peaks appears at the same interval as the upper limb weight peak. Because the right foot is attached to the block, when weight is applied to the right foot, little weight is applied to the left foot. Therefore, the left upper limb will be heavily loaded at that time. Additionally, it can be seen that when the weight is applied to the left foot, the right upper limb weight is lighter.

Considering the graph of the moving distance in terms of the time required, the moving distance was $3 \mathrm{~m}$, and it was found that the required time varied depending on the conditions. From the graph of the moving distance and acceleration for $3 \mathrm{~m}$, in normal walking it was found that it took about $3.5 \mathrm{~s}$ in seven steps. In contrast, in asymmetric walking, it was found that it took about $3 \mathrm{~s}$ in six steps. The shorter time is thought to arise because the stride was wider in the latter test. Because the $4 \mathrm{~cm}$ block was attached to the sole of the right foot, the leg length became longer. The upper limb weight then increased by the increment of the target value.

Next, it can be seen that the waveform features did not change much depending on the target value of $r U W$ for each condition, and there was no significant difference in the appearance of the waveform under each condition. This suggests that gait changes due to rehabilitation effects can be confirmed even if rehabilitation is performed by changing the load with different target $r U W$.

This way, it was also possible to calculate how much load was applied to the upper limbs, and it was confirmed that it was possible to visualize the load balance 


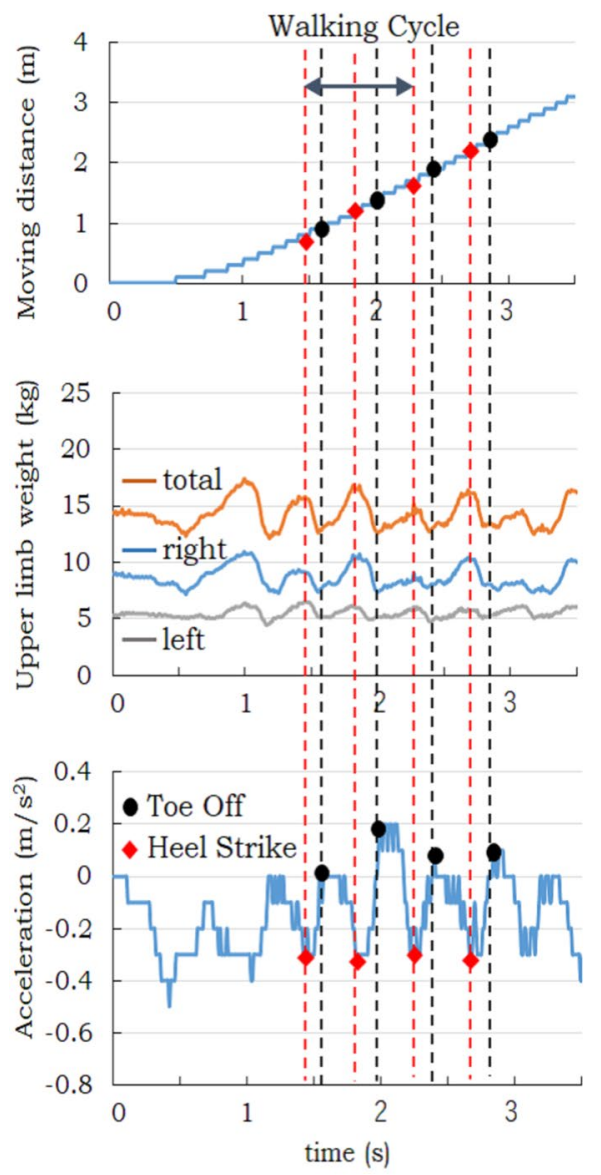

(a)Normal Walking

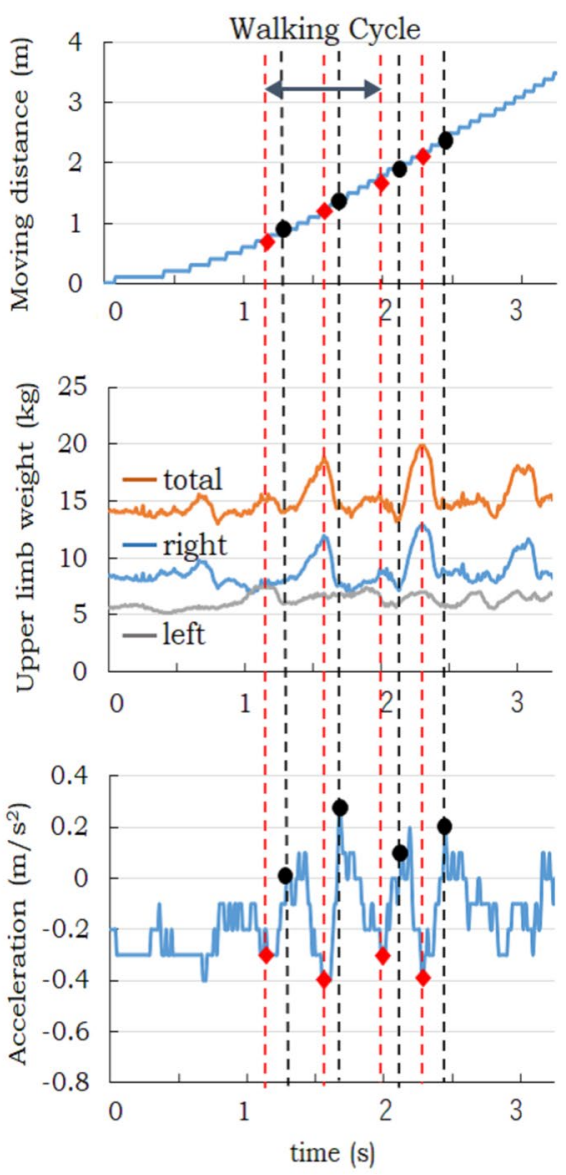

(b)Asymmetric Walking

Fig. 12 Measurement results under a target rUW of 30\%-a normal walking and $\mathbf{b}$ using the footwear with the attached $4 \mathrm{~cm}$ block on the sole of the right foot (to simulate asymmetric walking)

according to the gait by checking the acceleration value simultaneously. Utilizing this fact, it is suggested that there is a possibility of effective rehabilitation by quantifying the exercise information of rehabilitation, which is qualitatively applied in our device and method.

\section{Conclusions}

In this research, for developing a gait-training measurement system that enables effective rehabilitation training using IoT and measurement technology actively, we proposed a layer-based IoT measurement system which was implemented on a rolling stand-up walker.

In this paper, we developed measuring system modules about upper limb weight, acceleration (Module A) and moving distance (Module B). In consideration of versatility and convenience, these modules are able to have been implemented to a commercial rolling stand-up walker. Each measurement data can be viewed via web access. It was confirmed that upper limb load, acceleration and gait distance can be measured normally by using each of the mounted measuring modules, and it was possible to visualize the load balance according to the gait by simultaneously checking the acceleration value. So we can understand the exercise information of rehabilitation. By utilizing this fact, it is suggested that there is a possibility of effective rehabilitation by quantifying the rehabilitation which has been qualitatively applied to so far. In the future, multiple gait training devices will be operated at the same time in the hospital, and achieve improved measurement accuracy. At the same time, we will use data communication with the data server on the in-hospital LAN and try to make rehabilitation training and exercise information of daily life collectively manage to make rehabilitation more effective.

We developed measuring system modules that measured upper limb weight, acceleration (Module A), and moving distance (Module B). All measurement data could be viewed using internet. It was confirmed that 


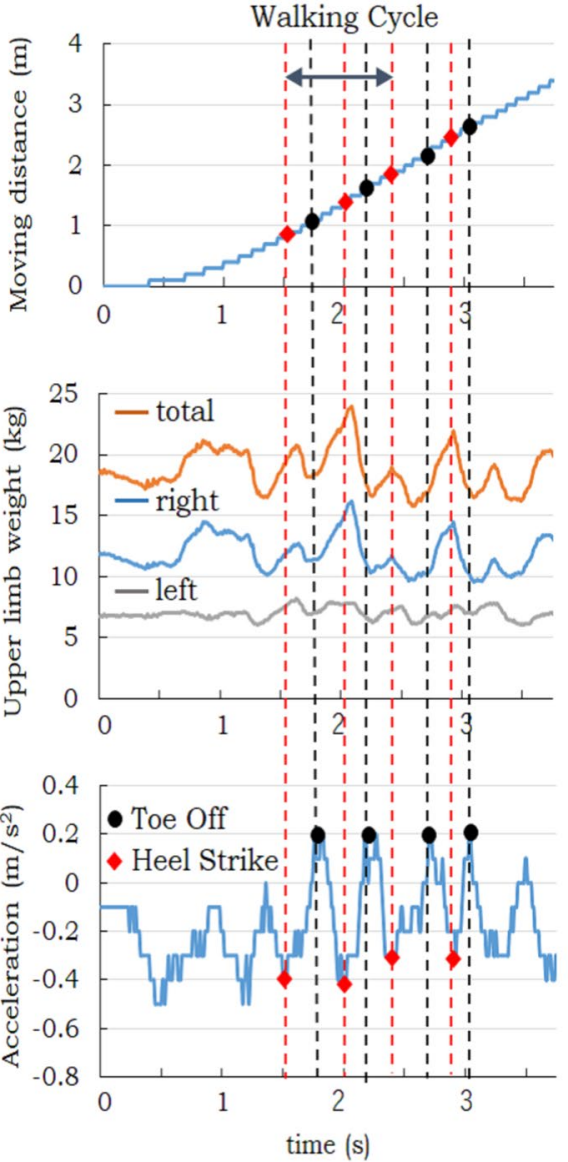

(a)Normal Walking

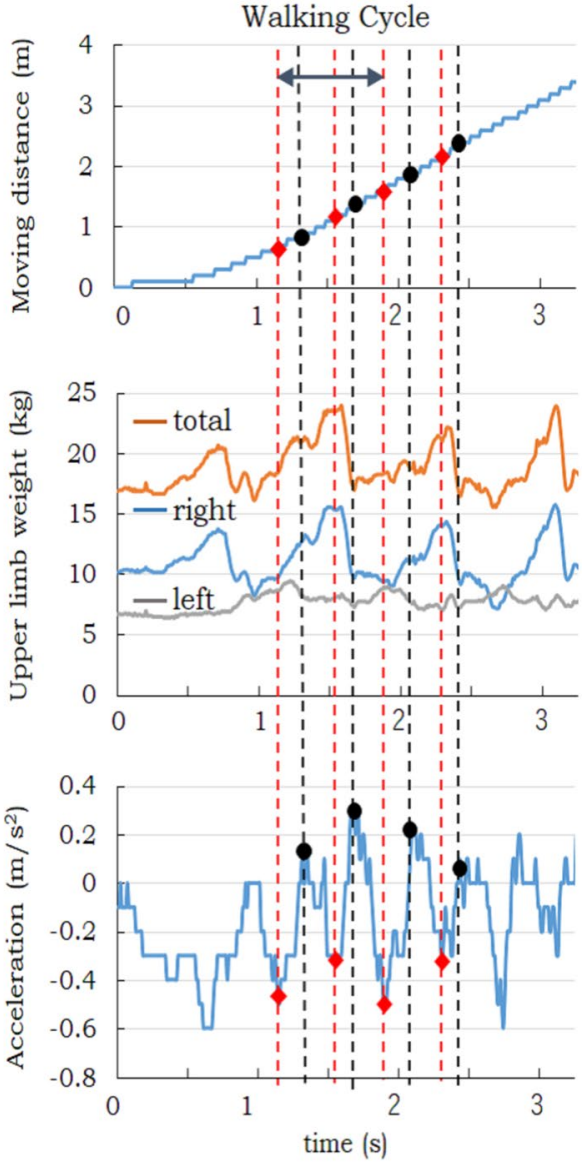

(b)Asymmetric Walking

Fig. 13 Measurement result for a target rUW of 40\% - a normal walking and $\mathbf{b}$ using footwear with the $4 \mathrm{~cm}$ block on the sole of the right foot

the upper limb load, acceleration, and gait distance could be measured normally using each of the mounted measuring modules, and it was possible to visualize the load balance according to the gait by simultaneously checking the acceleration value. This approach allows the user to understand and adjust the exercise information of rehabilitation. Utilizing this fact, it is suggested that there is a possibility of effective rehabilitation by quantifying the rehabilitation that has been qualitatively applied to so far. In the future, multiple gait training devices will be operated at the same time in the hospital, to achieve improved measurement accuracy. Simultaneously, we will use data communication with in-hospital LAN data servers to try and make rehabilitation training and exercise information of daily life more effective.

\section{Acknowledgements}

We appreciate the help received from Dr. H. Hori and Dr. J. Okada through valuable discussions held, and we thank the students of Osaka
Electro-Communication University Jeong Laboratory, Ogawa Laboratory, and Aino University Aoyama Laboratory for their help and support.

\section{Authors' contributions}

SJ led and oversaw the project. KO created and drafted the measurement system design and development. $\mathrm{KU}$ and IC developed and implemented the measurement modules. KU developed the network application. HA and $\mathrm{KY}$ participated in the discussion on system development based on physical therapy findings. All authors have read and approved the final manuscript.

\section{Funding}

This work was supported by the Graduate School Joint-Research Fund of Osaka Electro-Communication University.

\section{Availability of data and materials}

The datasets supporting the conclusions of this article are included within the article.

\section{Competing interests}

The authors declare that they have no competing interests.

\section{Author details}

${ }^{1}$ Department of Electro-mechanical Engineering, Osaka Electro Communication University, Osaka, Japan. ${ }^{2}$ Department of Physical Therapy, Aino 
University, Osaka, Japan. ${ }^{3}$ Department of Physical Therapy, Osaka Yukioka College of Health Science, Osaka, Japan.

Received: 7 October 2019 Accepted: 15 June 2020

Published online: 22 June 2020

\section{References}

1. Kondo Katsunori (2004) Kunrenryou to rehabilitation no kouka. Jpn J Rehabil Med Jpn Assoc Rehabil Med 41(12):849-853

2. Nagai $S$ et al (1989) Relationship between the intensity of stroke rehabilitation and outcome: a survey conducted by the Kaifukuki Rehabilitation Ward Association in Japan (second report). Jpn J Compr Rehabil Sci Syst Kaifukuki Rehabil Ward Assoc 23(4):123-145

3. Maruyama Hitoshi (2004) Trends in Rehabilitation for Elderly. Rigakuryoho Kagaku 19(3):163-167

4. "Ministry of Health, Labour and Welfare Official Site", http://www.mhlw. go.jp/file/05-Shingikai-10801000-Iseikyoku-Soumuka/0000120212_6.pdf Accessed 29 May 2019)

5. Akezaki Yoshiteru, Yamasaki Hiroshi, Nomura Takuo, Yoshimoto Yoshinobu, Yoshimura Susumu, Hamaoka Katumi, Nakata Hiroshi (2006) Weight bearing ratio on affected lower extremity which is necessary for walking independence in hemiparetic patients. Bull Kochi Rehabil Inst 8:27-31

6. Murata Shin, Otao Hiroshi, Murata Jun, Horie Jun, Yakihara Sachiko, Kai Kenichiro, Otsuka Makoto (2010) Relationships between the lower extremity function of the frail elderly and the timed up-and-go test and walking speed Rigakuryoho Kagaku 25(4):513-516

7. Nishimori Chisa, Yamasaki Hiroshi, KAwabuchi Masataka, Matsumura Fumio (2006) The measurement of maximum weight bearing value on lower extremity in hemiparetic patients. Bull Kochi Rehabil Inst 12:25-27

8. Shimada S, Sugiyama K, Ohtsuka S, Sawada Y, Arai M (2000) Training support system of the load control gait in rehabilitation. IEICE Tech Rep Human Commun Sci IEICE Tech Rep Human Commun Sci 100(288):17-24
9. Fuse Yasufumi, Hirao Keizo, Toriura Tetsuya, Kato Tomokazu (2019) Development of the load control device for rehabilitation of the bone fracture patient. Life. 2019, 3-3-3-1, Tokyo

10. Shimada Satoshi, Sugiyama Keisuke, Ohtsuka Sakuichi, Sawada Yoshinori, Arai Masanobu (2000) Training support system of the load control gait in rehabilitation. Inst Image Inform Telev Eng 24(51):17-24

11. Fukutama Kei, Ikeuchi Hidetaka, Abe Isao (2019) A study on screen presentation method for walking training system with foot load audio-visual feedback mechanism. Life. 2019, 3-3-3-3, Tokyo

12. Fukuyama K, Ikeuchi $H$ (2016) Construction of gait training system having audiovisual feedback mechanism by real-time presentation of the foot load -The output characteristics of the load in the gait paths ? Life 2016:482-485

13. WANG Yina, WANG Shuoyu (2015) Development of motion control method to collaborate with user's walking state for a walking support robot. LIFE. 2015, 1B2-08, Fukuoka

14. Uegami Kenji, Jeong Seonghee (2018) Evaluation of Indoor Walking Support Device with Upper Rotation mechanism and Viscosity Changeable Break System. 2018 JSME Conference on Robotics and Mechatronics, 2P2H03, Kitakyushu

15. PARAMOUNT, "Walking Assist Device KA-391", https://www.paramount. co.jp/product/detail/index/30/P0002252 Accessed 29 May 2019

16. Ogawa Katsushi, Jeong Seonghee (2017) Development of measurement system for robotic walking training device to improve exercise effect in convalescence rehabilitation. 17th International Conference on Control Automation Systems, pp.1939-1940

17. Okada Jun, OGAWA Katsushi, Jeong Seonghee (2018) Clinical significance of upper limb dependence measurement in walking training. Life 2018, $1-1-2$

\section{Publisher's Note}

Springer Nature remains neutral with regard to jurisdictional claims in published maps and institutional affiliations.

\section{Submit your manuscript to a SpringerOpen ${ }^{\circ}$ journal and benefit from:}

- Convenient online submission

- Rigorous peer review

- Open access: articles freely available online

- High visibility within the field

- Retaining the copyright to your article

Submit your next manuscript at $\boldsymbol{\nabla}$ springeropen.com 